\title{
Strategies for maintaining emotional stability: The case of nurses in private health care industry
}

\author{
Pushpika Subhashinie Mullakanda and Kumudinei Dissanayake*
}

${ }^{a}$ Commonwealth Executive Master of Business Administration Degree Program, Open University of Sri Lanka, Nawala, Sri Lanka ${ }^{b}$ Department of Management and Organization Studies, Faculty of Management and Finance, University of Colombo, Colombo 3, Sri Lanka

\begin{tabular}{|c|c|}
\hline CHRON I C L E & A B S T R A C T \\
\hline $\begin{array}{l}\text { Article history: } \\
\text { Received March 5, } 2015 \\
\text { Received in revised format } \\
\text { August } 162015 \\
\text { Accepted October } 132015 \\
\text { Available online } \\
\text { October } 152015\end{array}$ & $\begin{array}{l}\text { This study examines the emotional intelligence (EI) of nurses by differentiating inter-personal } \\
\text { and intra-personal dimensions, and explores the measures taken by them to maintain emotional } \\
\text { stability. Further, it investigates the relationship of demographic factors (age, gender and year } \\
\text { of experience) of nurses with their emotional stability. The case organization is a well } \\
\text { performing private hospital located closer to the metropolitan city. Study collects data through } \\
\text { both quantitative (survey) and qualitative means (discussion, observation, and interviews) }\end{array}$ \\
\hline $\begin{array}{l}\text { Keywords: } \\
\text { Emotional intelligence } \\
\text { Emotional stability } \\
\text { Nurses } \\
\text { Private health care industry }\end{array}$ & $\begin{array}{l}\text { using a sample of } 40 \text { nurses selected conveniently from different ranks. The study finds that } \\
\text { the level of EI of nurses in case hospital is slightly high, and their inter-personal skills are } \\
\text { higher than intra-personal skills. They maintain emotional stability by means of emotion gaps, } \\
\text { emotional shifts and sharing. Findings suggest that both inter-personal and intra-personal } \\
\text { dimensions of EI will not be equally important for nurses in dealing with patients, and the level } \\
\text { of EI required in the job is not equal among different ranks of nurses. }\end{array}$ \\
\hline
\end{tabular}

\section{Introduction}

Emotional intelligence (EI) has become important not only for achievements in personal life but also in improving performance at work (Naseer et al., 2011). Accordingly, EI has become a key term in management consultation, leadership training (Goleman, 2001), organizational performance (O’Boyle et al., 2011), entrepreneurial performance (Cross \& Travaglione, 2003), and career development too. As Naseer et al. (2011) notes, EI is critical to high performance, due to the fact that it helps a person to stay motivated even under stressful circumstances, motivates peers and subordinates, deal with complex inter-personal relationships successfully. Theoretically seen, EI is not a uni-dimensional concept, but instead stands two-dimensionally, evolving around concepts directed towards self- and others, thus involving individual emotions and cognitions (Goleman, 2001).

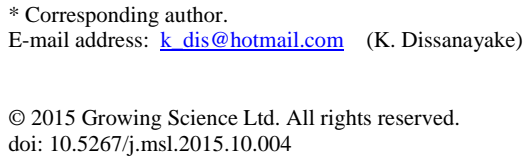


Employees, engaging in boundary spanning roles and located at the interface with customers or at the direct interaction with external stakeholders in organizations, need to play a vital role in managing their emotions. This may be for personal impression management (Rosenfeld et al., 1995) or due to demands of emotional labor (Luthans, 2008) at work. With these demands, health care organizations, among other service-based firms, become emotion-driven workplaces. Further, health administrators and their employees are required to contend with providing quality service to their customers during a period of limited physical and human resources. In addition, it is very competitive and standards of the industry are getting better day by day due to this intense competition.

Management and organization research looks into the ways of improving the level of EI of employees, aligning with organizational objectives. Relations between emotions and leadership (Kent, 2006), emotions, team empowerment and proactivity (Erkutlu \& Chafra, 2012), and measuring EI of leaders (Groves et al., 2008) evidence to the existing knowledge which informed the significance of emotionsrelated findings in organizational research. Thus, scholarly works on EI from managerial and organizational perspectives are not rare or limited. However, despite a handful of attempts made to investigate how EI works in nursing context (e.g., Brenda \& Louis, 2002; McQueen, 2004), an investigation into the attempts made by nurses themselves in improving their EI has not been well reported in the existing literature. Further, the investigation of demographic factors such as age, job ranks, gender, years of experience, level of education, etc. of nurses in the private health care industry would predict some interesting links, and possibly would suggest some valuable clues for designing their training programs with a focus on improvement of EI. Nonetheless, this knowledge is yet to be created which is recognized as an empirical gap in the given context.

In Sri Lanka, both public and private sectors cater to the health needs of people while competing with each other. It is interesting to note that both the sectors are highly concerned about meeting their service standards. Thus, paying a closer attention to maintain the service quality of nurses has been a key success factor in health care industry in Sri Lanka. On these grounds, an outstanding private hospital is selected for the present study for probing into the measures taken by nurses for maintaining their emotional stability at work.

Accordingly, purpose of this study is to investigate the emotional intelligence, strategies used for maintaining emotional balance, and the effect of demographic factors of nurses by means of a case study. Thus, while examining the level of EI, it first differentiates inter-personal and intra-personal dimensions of EI of nurses. Next, it explores the strategies used by nurses to maintain their emotional stability. Finally, it investigates the effect of demographic factors (age, gender, year of experience, etc.) on maintaining emotional stability by nurses. This paper is organized as follows. Followed by this introduction, it reviews the literature related to the key areas of the topic. Presenting the method adapted, we discuss the major findings of the study. Finally, we conclude with the results while discussing the implications and avenues for further research.

\section{Review of Literature}

\subsection{Emotional Intelligence}

Emotional intelligence is the ability to manage oneself and one's relationship in mature and constructive ways (Kinicki \& Kreitner, 2009). It has two intra-personal domains; self- awareness and self-management, and two inter-personal domains; social awareness and relationship management (Goleman, 2001).

Emotional Intelligence is often referred also as Emotional Intelligence Quotient (EQ) which is the ability of an individual to perceive, assess and manage emotions of his/her own self and of other people. Mayer and Salovey (1993) define EI as the ability to monitor one's own and others' feelings and 
emotions, to discriminate among them and to use this information to guide one's thinking and actions. Thus, EI has four main components, i.e. the ability to:

1. perceive self-emotions

2. utilize these emotions to guide self-thinking and actions

3. understand emotions of others

4. manage emotions to achieve goals

Further, it is argued that EI can be nurtured and developed (Luthans, 2008). The source of EI of an individual is identified to be the communication between his/her emotional and rational brains. Thus, EI starts in the brain, and it activates before a person do think rationally. It is proved that people have emotional reactions to events before his/her rational mind engages in the event.

\subsection{Domains of Emotional Intelligence}

Mayer et al. (2003) identified four skills of EI which includes emotional awareness (own and others), emotional management (own and others), emotional understanding and emotional facilitation. These four skills are easily recognized in two dimensions; interpersonal and intrapersonal intelligence, and divided into four domains. Thus, the theory recognizes two intra-personal domains (self-awareness and self-management), and two inter-personal domains (awareness of others/empathy and relationship skills) (Morrison, 2006). Figure 1 below depicts the two dimensions and four domains of EI. The arrows denote the directions of relationships among each.

\section{Intrapersonal Intelligence}

Self-awareness

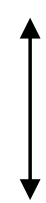

Self-management

\section{Interpersonal Intelligence}

Social awareness

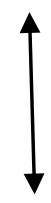

\section{Relationship management}

Fig. 1. Four domains of EI

Accordingly, EI comprises two main abilities of an emotionally intelligent individual, such as (a) appraisal and expression of emotions of own and others, and (b) use of emotions and emotional management of own and others.

Extant literature in the area of management and organizations has looked into the relationship between EI and several organizational processes. EI and the leadership in organizations have been accorded scholars' attention to a larger extent (e.g., Dulewicz \& Higgs, 2003; Fineman, 2000; Groves et al., 2008; Palmer et al., 2001; Pescosolido, 2002; Rahim et al., 2006). Relationships between EI and Organizational behavior and certain behavioral outcomes too have been investigated further (e.g., $\mathrm{Al}$ Hajj \& Dagher, 2010; Carmeli, 2003; Rahim \& Malik, 2010).

\subsection{Emotional Intelligence in Nursing}

Emotions and nursing work is an area researched in the health care industry (e.g., McQueen, 1997; McQueen, 2000; McQueen, 2004; Meyer, Fletcher, \& Parker, 2004; Smith, 1992). According to Goleman, (as cited by McQueen, 2004), there are four separate abilities within interpersonal intelligence, such as, the skills in forming groups appropriately, negotiating possible solutions for 
problems, connecting personally and engaging in social analysis. Thus, fostering interpersonal intelligence in nursing has been recognized in the existing literature. The interpersonal intelligence is an essential part of nursing work as it needs nurses to understand how patients feel, thereby demonstrate empathy in their care (McQueen, 2004). Further, nursing work involves group work, thus necessitating team-work, negotiating, and organizing skills for successful performance at health care industry. Managing emotions in organizations enable the organizing function (Fineman, 2000; Mark, 2005). Pescosolido (2002) asserts that leaders are managers of group emotions. According to his explanation, leader emergence and leader success can be subject to leader's degree of empathy, group norms of emotional expression, and ambiguous feedback regarding the group's performance. Thus, EI plays a vital role in deciding leader success at group work.

The management of emotions or maintaining emotional stability enables an individual to join or unjoin him/herself from an emotion in a given situation. It further enables the individual to control his/her immediate reactions in the particular situation (Naseer et al., 2011). When patients are hospitalized for shorter periods of time, there is a need for hospital nurses to be able to form good rapport rapidly with patients. This is necessary for the development of trusting relationships, so that patients feel able to discuss personal, sensitive issues associated with their recovery. Thus, EI of nurses becomes significant factor in health care industry. Thus, care cannot be confined to the physical element but also the psychological and spiritual needs (The Journal of Nursing, 2007).

Existing literature have investigated the impact of EI on recruitment of nurses (e.g., Cadman \& Brewer, 2001), training of nurses (e.g., Evans \& Allen, 2002; Secor et al., 1999) and nursing leaders (e.g., Vitello-Cicciu, 2002) at workplaces.

\subsection{Demographic Factors- Gender and Marital Status}

Demographic factors have significant impact on emotional stability of people. As per Singh (2003), women are more emotionally stable than men due to the high levels of EI of the former. Further, he finds that there is no significant correlation between marital status and EI of individuals. His analysis shows that with growing age, a person's emotional stability increases, but after reaching a peak it starts declining thereby creating proportionate relationship between EI and leadership of employees. Sarinnapakorn and Sucaromana (2013), investigating the level of EI of business consultants in Thailand, found no significant difference between levels of EI based on gender and years in the industry, even though it was at a high level. Thus, gender, age, and work experience have shown inconsistent relationships at different research contexts. It may be interesting to see the level of EI and the emotional stability of nurses in private health care industry.

\section{Methods}

With the intention of conducting the investigation deeply in to the ground realities of EI dimensions and the particular measures taken by nurses, this study adapted single case study strategy as proposed by Yin (2009). The study was conducted in ABC Hospital which is a Private Hospital in Colombo, Sri Lanka. It is a large Hospital, which employs state of the art technology to serve the customers.

\subsection{Research Setting}

This hospital has been able to develop a unique culture over five years from its operations, which has a lot of influences on service excellence and training and development of Human Resources. Currently they manage their own Nurses' Training School in-house. The first batch of nurses are already been recruited and the second batch is being trained to provide nursing qualification and intensive training. 
They would be qualified and absorbed to the hospital eventually. Nurses' cadre is 128 in this hospital under four grades as follows.

Table 1

Nurses’ Cadre at ABC Hospital

\begin{tabular}{lcc}
\hline Grade & Cadre \\
\hline Grade 1A & 40 \\
Grade 1B & 1 \\
Grade 2A & 9 & 9 \\
Grade 2B & 93 \\
Grade 3A & 13 \\
Grade 3B & 3 \\
Grade 4 & 128 \\
$\quad$ Total & 53 \\
\hline Source: Survey data (2013) & & \\
\hline
\end{tabular}

There are 10 sections at ABC Hospital; identifiably, Ward 1 \& 2 (Surgical \& medical), Ward 3 (Gynecology \& observation), Ward 4 (Pediatric), Intensive Care Unit (ICU), Out Patient Department (OPD), Day Treatment Unit (DTU), Surgical Step Down (SSD) and the Theatre. These are managed by eight Nursing-In-charges under the supervision of the 'Head of Nursing' of the Nursing Department (See Fig. 2 below).

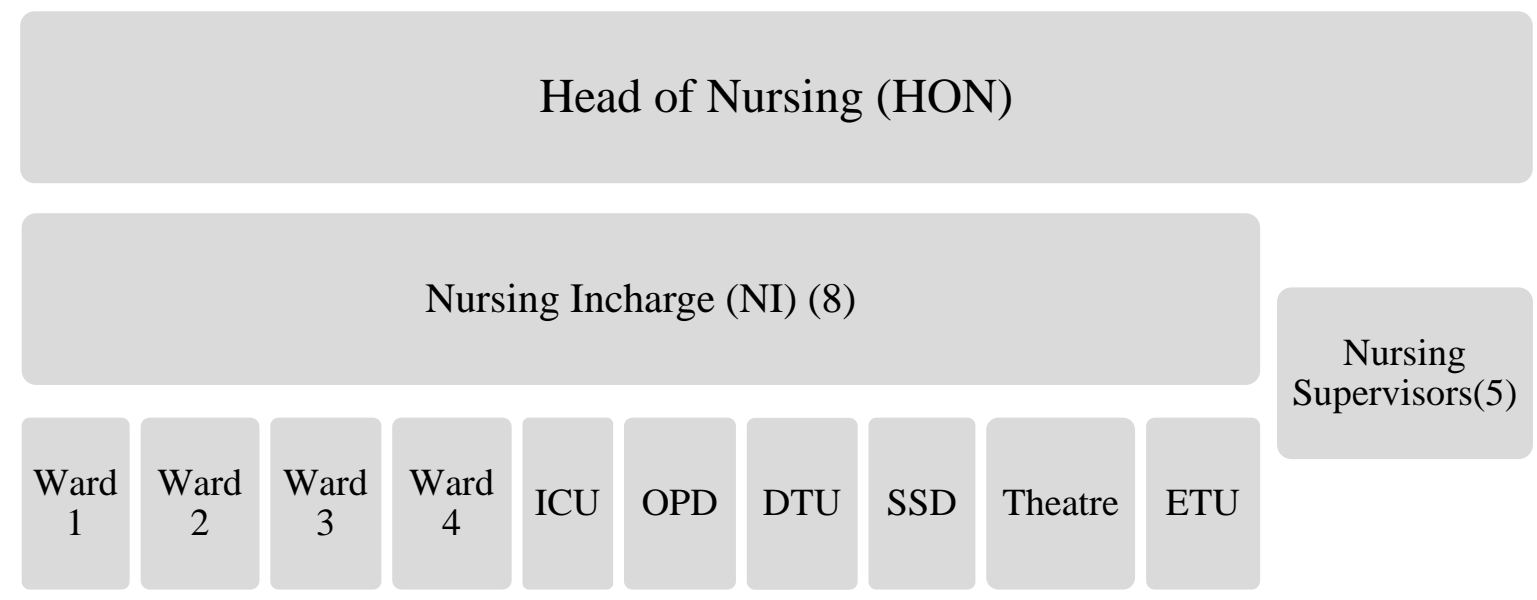

Fig. 2. Structure of Nursing Department

\subsection{Data Collection Protocol}

Data were collected through both quantitative and qualitative surveys and from primary and secondary sources. Primary data sources were discussions, interviews, surveys, and observations.

Discussions: After obtaining permission from the hospital management to conduct the research study, preliminary discussions were conducted with the Human Resource Manager and the Head of Nursing for the researchers to be familiar with their HR and patient care policies and practices and their expectations in order to have an insight into their culture and working practices to set the ground for the researcher to move freely in meeting nurses in the Hospital. Further discussions were conducted with Head of Nursing and Nursing-in-charges (NI) in order to get an understanding of the teams in different sections and their sub-cultures.

Interview: The primary data were gathered through face to face interviews with a sample that consisted of conveniently selected 40 nurses from seven selected sections of the hospital (representing Grades 1 , 2 and 3). Each nurse was interviewed and data were recorded by the main researcher her-self. Further, 
ten patients, their family members, and peers were interviewed in order to evaluate the EI and performance levels of nurses.

A structured questionnaire was used as the guide to interviews in order to make sure the consistency of data collection at individual level. This questionnaire covered the four domains of EI: (a) selfawareness, (b) self-regulation, (Intra-personal dimension) and (c) social awareness, and (d) social skills (Inter-personal dimension) (Goleman, 1995). This instrument was a pre-prepared, pre-tested guide for interviews. It consisted of two parts; Part A, and Part B. Part A of the questionnaire focused on individual nurses and it covered demographic data including the age, gender, years of experience in the industry, etc., together with open ended questions that allowed reporting participants thoughts and feelings about their emotions and measures. Part B of the questionnaire was focused on Nursing-InCharge (NI) in order to get a feedback/response on nurses working under him/her. Two main dimensions of emotional intelligence, i.e. understanding and managing self-emotions (intra personal relationship) and understanding and managing others' emotions (inter-personal relationship) were reported by the researcher on a 5 -point Likert scale (from $5=$ High to $1=$ Low) in the second part of the Part $\mathrm{B}$. The areas probed into were:

(1) Managing stress,

(2) Dealing with peers, and

(3) Appreciation by clients.

Results obtained on this scale were recorded and interpreted as follows: 3 and above $=$ high EI, and below 3 = low EI.

Observation: Observation of interactions of nurses with their superiors, peers, patients and even family members of patients were done before commencing the interviews. This helped understand the level of consciousness and management of emotions by nurses. Further, the researcher took necessary steps in observing the reactions, facial expressions and gestures of nurses, while conducting the survey/interviews, so that she may be able to interpret and analyze their responses easily. Nurses were further observed to see the manner they talked to patients and family members of patients, the way they inserted and removed cannula, how they provided medicine and helped with injections, how they transported patients to and from theater and other wards/sections, etc. for understanding nurses' intrapersonal and interpersonal skills.

\section{Secondary Sources of Data}

Internal records of ABC Hospital were used to collect information on structure of nursing department, nurses' cadre, nurses’ profile details, etc. in designing the data collection protocol and the instrument.

\subsection{Sample Profile}

A sample of conveniently selected 40 nurses out of the population of 128 nurses, were used for the study. The structure of the sample is presented in Table 2 as follows.

\section{Table 2}

Job rank representation of nurses in the selected sample

\begin{tabular}{cccc}
\hline Grade of nurses & Selected nurses & Percentage of selected nurses & Nurses in the cadre \\
\hline Grade 1 & 13 & $31.7 \%$ & 41 \\
Grade 2 & 9 & $50.0 \%$ & 18 \\
Grade 3 & 18 & $27.3 \%$ & 66 \\
Grade 4 & 0 & $0 \%$ & 3 \\
\hline Total & 40 & $31.25 \%$ & 128 \\
\hline
\end{tabular}

The representation of selected 40 nurses from different sections of the hospital is presented in Table 3 as follows: 
Table 3

Section representation of nurses in the sample

\begin{tabular}{cccc}
\hline Section & Selected nurses & Percentage (\%) & Nurses in the cadre \\
\hline Ward 1 & 6 & 54.55 & 11 \\
Ward 2 & 12 & 80.0 & 15 \\
Ward 3 & 6 & 40.0 & 15 \\
Ward 4 & 3 & 16.67 & 18 \\
ICU & 8 & 50.0 & 16 \\
OPD & 0 & 0.0 & 1 \\
DTU & 0 & 0.0 & 7 \\
SSD & 0 & 0.0 & 15 \\
ETU & 2 & 13.3 & 12 \\
\hline Theatre & 3 & 25.0 & 128 \\
\hline Total & 40 & 31.25 &
\end{tabular}

Interviews, observation and data collection was carried out in four wards (i.e., two Medical and Surgical wards, Pediatric ward, Gynecology ward), Theatre, Emergency Treatment Unit (ETU) and Emergency Care Unit (ECU).

\section{Findings and Discussion}

\subsection{Demographic Background of the Sample}

Demographic factors of the sample were analyzed in terms of age, gender, home-town, level of experience, marital status, number of children, etc. Findings are as follows:

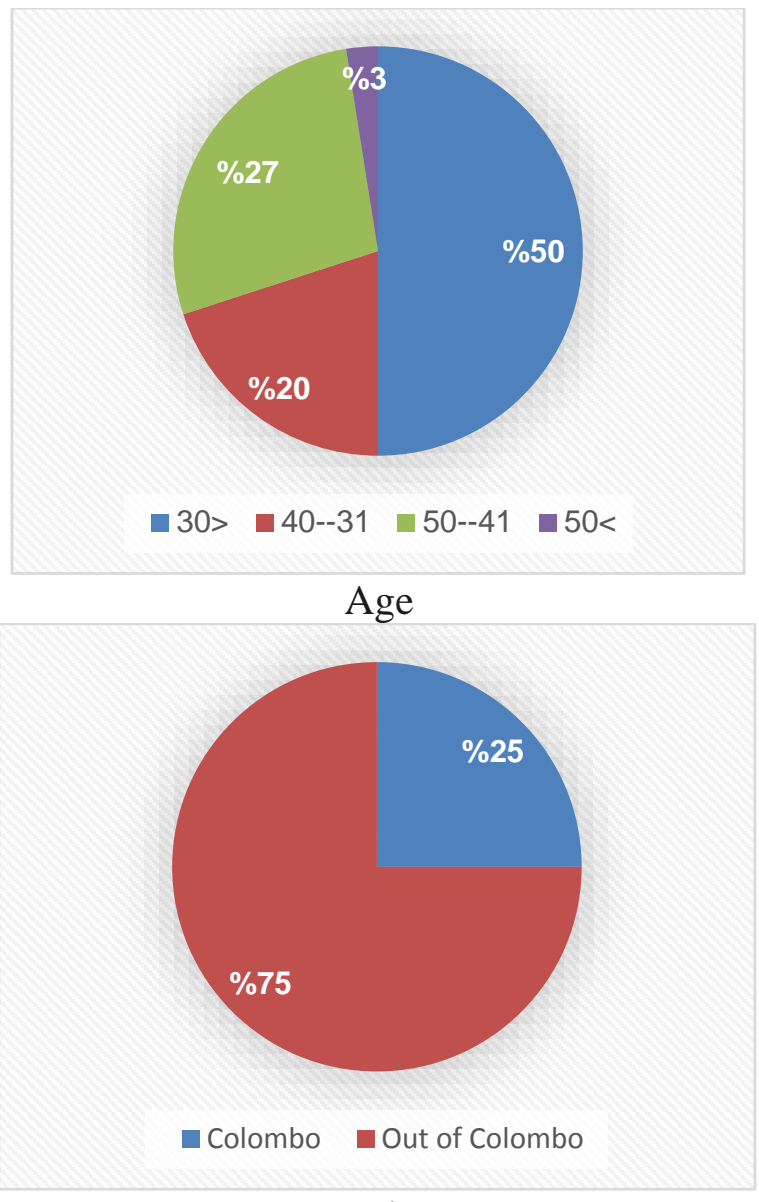

Expertise

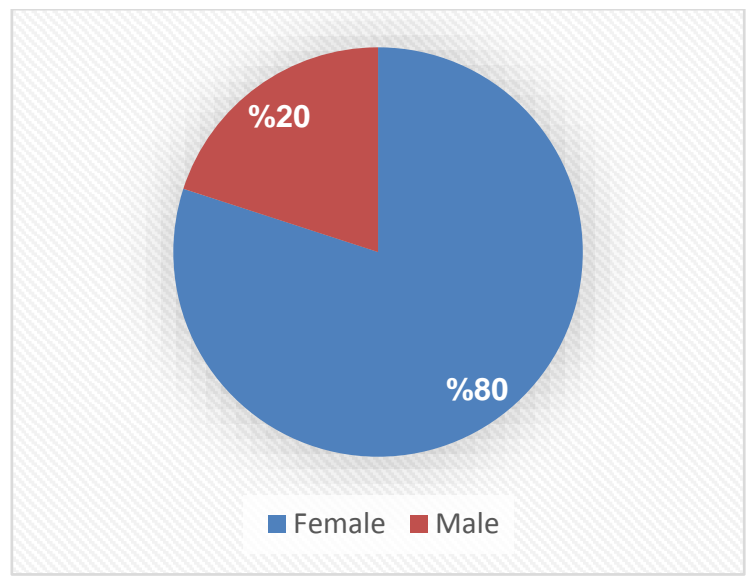

Gender

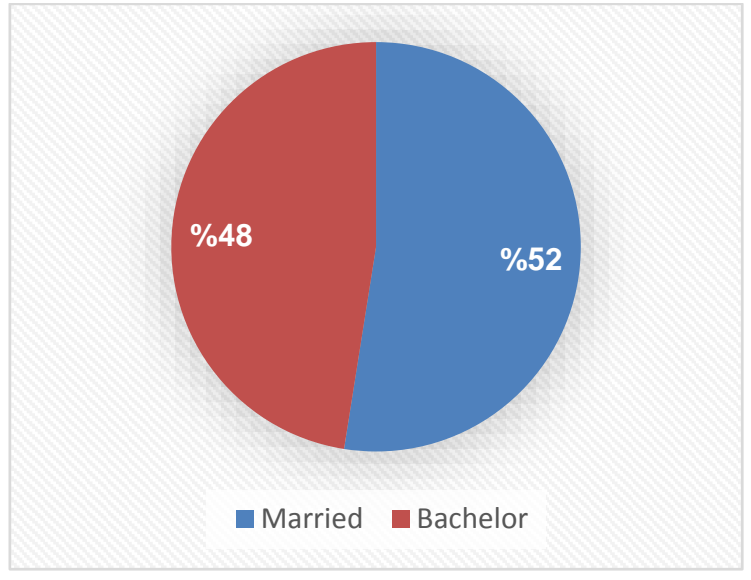

Marital status 


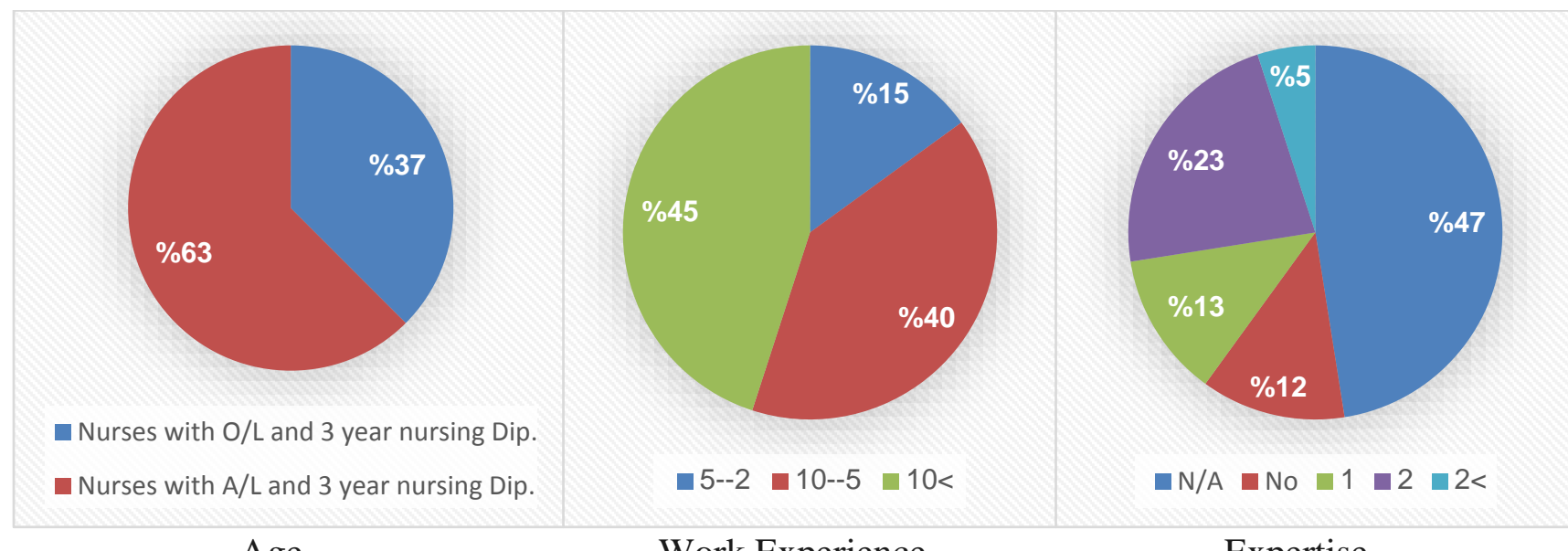

Age

Work Experience

Expertise

Fig. 3. Personal characteristics of the participants

Average age of nurses in the sample was 34 and $50 \%$ of them were below 30 years which means majority was young. $80 \%$ was female and $75 \%$ of them are from out of capital city. Sixty two point five percent of nurses had been qualified with A/L and 3 years Nursing Diploma, while $95 \%$ of Nurses had above 5 years of experience at work where the average experience of them is 11 years (depicting a high standard of skill level of them). Since the majority is young, $47.5 \%$ is bachelors. Also $47.5 \%$ of married nurses do not have children.

\subsection{Effect of demographic factors (age, gender and year of experience) on maintaining emotional stability by nurses}

In the present study there were some interesting relationships found between demographic factors and emotional intelligence of nurses. That was the positive relationship between these two variables. E.g., majority of nurses who scored more than 14 on five point Likert scale possessed more than 10 years of work experience in the same job. In evaluating the relationship between marital status and EI, it was found that three unmarried nurses in the sample who were above 40 years of age had their rating below 11 on five point Likert scale.

As per the explanations on open ended questions and observations, it is observed that some demographic factors such as years of work experience, marital status, have positive impacts on emotional stability of nurses. When further investigated, those who had long-standing experience had undergone intensive training, and got matured over a period of time, which enabled emotional stability.

\subsection{Level of Emotional Intelligence of Nurses}

\section{Table 4}

Level of EI based on superiors' comments

\begin{tabular}{lccccc}
\hline Dimensions & Sample population & Minimum & Maximum & Mean & Std. Deviation \\
\hline Managing stress & 40 & 2.00 & 5.00 & 3.6000 & .74421 \\
Dealing with peers & 40 & 2.00 & 5.00 & 4.1750 & .84391 \\
Appreciation by clients & 40 & 2.00 & 5.00 & 3.9750 & .89120 \\
\hline
\end{tabular}

As per the ratings marked on the five-points Likert scale by superiors on 'Stress Management' of nurses in the case hospital, the mean value was recorded as 3.6. This means that the nurses of the selected hospital have a satisfactory level of stress management. (Standard deviation $=0.74$ ). The mean value of 'working with peers' of nurses was reported as 4.17 (with a standard deviation of 0.84).Similarly, 'customer appreciation' of nurses reported a mean value 3.97(with a standard deviation of 0.89). Meanwhile, the lowest and highest values for the three constructs, Stress Management, working with peers, and customer appreciation, were two and five. 
As per the analysis of ratings given by superiors on Likert scale, mean value of all the ratings on whole sample is 3.92. This is located in the 'high' category but closer to lowest level (High=3 and above). Thus, the empirical findings prove that the EI level of nurses is fairly high in ABC Hospital. On the other hand, the section-wise highest EI was recorded in Ward 2 and Theatre, with an average score of a nurse 12.83 and 14.33 respectively. Thus, the nurses in these two sections showed high EI as a group than the groups in other sections.

In differentiating inter-personal and intra-personal dimensions of EI of nurses, the mean values on ratings given on three constructs have been considered. Construct 1 that focused on intra personal dimension had a mean value of 3.6 and it implies that nurses' intra-personal dimension of emotions is also fairly high as it is located in the category 'high' on Likert scale. On the other hand, construct 2 and 3 that focused on inter personal dimensions had mean values of 4.17 and 3.97 (average =4.07) respectively. Hence they reported a very good sense of others' emotions and ability to manage them. Interestingly, this was again proved by the qualitative data, which revealed that 32 nurses out of 40 understand other nurses' emotions/stress through sensing. Thus, all these findings imply that these nurses have better inter-personal skills than intra-personal skills.

\subsection{Strategies used by Nurses to Maintain Emotional Stability}

Strategies used by nurses in managing their work were analyzed and themes were identified as follows:

Table 5

Strategies used by Nurses for Maintaining Emotional Stability

\begin{tabular}{llll}
\hline Measures & Freq. & \multicolumn{1}{c}{ Coding } \\
\hline Managing emotions at daily work & 7 & Ignore \\
Not taking things seriously & 8 & Control \\
Controlling by self & 5 & Share \\
Talking to somebody & 6 & Internalize \\
No stress/Taking it as part of job & 6 & bare \\
wait for a while/keep quiet to settle down & & \\
\hline Managing intensive emotions & 10 & Emotion gap \\
Not taking things seriously & 8 & Emotional shift \\
Listen to music/watch TV or movie/sleep & 4 & Bare \\
Spends time alone, quietly & 6 & Sharing \\
Telling somebody-spouse, parents, superior & 5 & Internalization \\
Job/work is a part of life, no stress & 2 & Controlling \\
Controls & & \\
\hline
\end{tabular}

As per the analysis of answers given for open-ended questions by the nurses, 'maintaining gap between emotions and self' was performed by the majority (10). 'Emotion shift' is the second highest measure taken by nurses of ABC Hospital in order to maintain emotional stability at work.

The observation of nurses in their attending to patients, interacting with patients and their family members, consultants and doctors, nursing in charges (NI), peers, etc. helped the researchers identify particular behaviors, which provided clues in understanding the way they attempted to manage their emotions, how they dealt with others at certain instances, and importantly, their gestures and facial expressions at times. 
- Elderly and unmarried nurses showed emotional imbalance at work.

- All become emotionally imbalanced at deaths of patients even for a while. However, less experienced nurses found it more difficult to balance their emotions at deaths.

- Corporate culture, support of top management, attitude towards staff training and developments has had numerous positive impacts on improvement of EI of nurses.

- Superiors' way of team management and personality seems to be very much important in managing and improving EI of the team.

- All the painful routine work of a nurse, such as inserting and removing cannula, injecting medicine, cleaning wounds, etc. have become habitual actions to the nurses, and so they did not become emotionally imbalanced at work. But, outside the work context, they were completely different and emotionally unstable.

- Nurses become emotional when situation resembles one of their own. E.g., at a death of an infant - a nurse with her own child at the same age level became more emotionally unstable and seemed the situation was unbearable.

- Those who have got intensive training, better exposure to learn on the job (government or private sector), and long-standing experience showed a lot of confidence and emotional balance at work.

\subsection{Discussion}

It was obvious in the study that the nurses have recognized some measures for their emotional stability through their experience and others advice. Hardly anyone look for any guidance from eastern or western psychology to improve inter-personal or intra-personal skills and emotional stability. Apart from teaching in western psychology, "Vipassana meditation" in Lord Buddha's teaching can be very useful in improve the mindfulness over thoughts, feelings and body in order to develop intra-personal domain as well as inter-personal domain. This is in line with Minda's (2013) recognition that practicing meditation (self-awareness and self-observation) can increase EI, sociability, empathy, happiness, joy, positive thinking and moral development, improve social relationships, and help management of negative emotions.

Previous research indicates that there is a positive relationship between EI and job performance of team members. As noted, when the team members are highly proficient at appraising and regulating their own emotions, it leads to less supervisory interference and high performance. However, when team members have low EI, they are noted to be less proficient at appraising and regulating their emotions, and thus they have to get assistance from their managers (Naseer et al., 2011). The present study found that there is a unique sub-culture in Ward 2 and Theatre that recorded high group EI. When the Nursing in-charges of these sections were interviewed we realized that they have had a unique leadership where the nurses were mentored gradually so that their EI would have been improved subsequently. Since EI can be developed (McQueen, 2004), the nurses would have been trained and developed in this unique team culture under the correct leadership of in-charges.

McQueen (2004) states that the communication between our emotional brain and the rational brain is the physical source of emotional intelligence. As such, emotional intelligence requires effective communication between the rational and emotional centers of the brain. Once the brain is trained by new strategies for emotional stability, subsequently, the emotionally intelligent behaviors of people may turn to habits. In the context of ABC Hospital, this theoretical stance became obvious. It was also supported by the relationship between years of experience and EI of nurses. Nurses with more number of years of experience potrayed high level of EI. Training and development they have gone through with the experience whould have made them matured and once the brain is trained, expected bahavior 
whould have become habits. The study observation justifies the previous speculations on the 'additive value of EI', which comes in the form of showing better interactive skills, are more co-operative and closer relationships by emotionally intelligent people in the long-term (McQueen, 2004).

As per Friedman and Riggio (cited in Morrison, 2006), the most emotionally expressive people transmit their mood to others around. Accordingly, a positive resonance occurs when two people's moods align around positive feelings; which create optimism, mental efficiency, fairness and generosity. In the present study, we found that highest EI was portrayed in Ward 2 and theatre. As per the observations, both the sections are under emotionally stable leaders. That would have set the grounds in the particular section to improve EI of nurses. The reason behind this could have been the positive resonance occurred when their moods align around positive feelings (Morrison, 2006). When the discussion turns to the demographic factors of nurses, we remark inconsistencies with previous findings. Thus, even though Singh (2003) asserts that women are more emotionally intelligent than men, present research did not find gender-based differences in EI, other than that on marital status and working experience at the context of ABC hospital. Research studies pertaining to factors related to nurse interactions with elderly people have shown that the educational level of nurses influenced nurse interactions with elderly patients. In contrast to this fact, there was no relationship established in the context of ABC Hospital to confirm this finding.

\section{Conclusions}

Emotional intelligence seems to be a more relevant concept in health care, when it is considered important for practitioners to understand patients' perspectives and for nursing leaders to engage in relationships that will facilitate successful management. Findings of observation prove that the successful management of Nurses-in-charge set the grounds for nurses working in their teams to maintain a high level of group EI. Emotional stability of these leaders played a vital role in this case.

Inter-personal skills and Intra-personal skills both have been recognized as needed for better performance in work organizations. In the present study it was observed that some demographic factors such as years of work experience and marital status have positive impacts of emotional stability of nurses. This proves that unlike IQ, EI can be developed through training and development over a period of time. When people are matured with experience, knowledge through training in latter part of their career or life span, EI can be developed. Demographic factors like, marital status and years of working experience having influence on emotional stability can have implications on development of recruitment policy of nurses. These demographic factors can be necessarily checked at recruitment.

Nurses have their own way of managing their emotions. Although they have not undergone any intensive training on EI they clearly know that it is a must to manage emotions to avoid 'burnout' situations therefore each and every nurse has his/her strategy to manage them on daily basis. Thus nurses in ABC Hospital maintain emotional stability by emotional shift, maintaining emotion gap and sharing, etc. in the nursing context.

\subsection{Implications}

Findings of this research study directs insights for the relevant case hospital to judge where they stand in terms of EI/EQ of nurses which is equally important as IQ for better service delivery. Based on the finding Hospital management can have an understanding of the impacts of corporate and sub-cultures, training and development on soft skills, etc. This has a variety of implications on training and development policy of ABC Hospital and other hospitals in the private health care industry. 
Also this study acknowledges the hospital management that they should consider demographic factors of nurses at the point of recruitment and selection. Accordingly this may extend implications on recruitment and selection policy of hospitals in private health care industry.

According to the findings of this study, and on contrary to the existing knowledge, it may suggest that both inter-personal and intra personal dimensions will not be equally important for nurses in dealing with patients. Thus the theory may be modified to accommodate the differences in different sectors, industries in relation to different stakeholder categories that they deal with (E.g., peers, clients, superiors etc.).

Further, hospitals in the private health care industry can organise training and development for both nurses and nurses in-charge to improve the level of EI for personal satisfaction and customer satisfaction.

\subsection{Limitations and Directions for Further Research}

Present study is not free from limitations. As in any other research study of this nature, this study was based on the information revealed by the nurses that can be subjective and biased. Also the views and comments of nurses with different family backgrounds may affect the consistency of findings. Also the feedback given by each superior on nurses is not standardized and thus this too may lead to inconsistency of views. Moreover, 360 degree appraisal of nurses was not able to conduct, but it was limited only to the appraisal of the superior due to time constraint and practical difficulties emerged from the part of case organization.

This study was based on a single case. Future research can focus on multiple cases selecting both private and public sector hospitals to cover a wider spectrum. That will enable the researchers to compare EI level of nurses in private and public sectors. Further studies can be continued in several industries to compare EI level of employees at multiple ranks and in different industries.

\section{References}

Al Hajj, R., \& Dagher, G. (2010). An empirical investigation of the relation between emotional intelligence and job satisfaction in the Lebanese service industry. The Business Review, 16(2), 7177.

Brenda, F. \& Louis, R. (2002). Emotional Intelligence: A Core Competency for Health Care Administrators. The Health Care Manager, 20(4), 1-9.

Cadman C. \& Brewer J. (2001) Emotional intelligence: a vital prerequisite for recruitment in nursing. Journal of Nursing Management, 9(6), 321-324.

Carmeli, A. (2003). The relationship between emotional intelligence and work attitudes, behavior and outcomes: An examination among senior managers. Journal of Managerial Psychology, 18(8), 788813.

Cross, B., \& Travaglione, A. (2003). The untold story: Is the entrepreneur in the $21^{\text {st }}$ century defined by emotional intelligence?. International Journal of Organizational Analysis, 11(3), 221-228.

Dulewicz, V., \& Higgs, M. (2003). Leadership at the top: The need for emotional intelligence in organizations. The International Journal of Organizational Analysis, 11(3), 193-210.

Erkutlu, H., \& Chafra, J. (2012). The impact of team empowerment on proactivity: The moderating roles of leader's emotional intelligence and proactive personality. Journal of Health Organization and Management, 26(5), 560 - 577.

Evans, D., \& Allen, H. (2002) Emotional intelligence: its role in training. Nursing Times 98(27), 4142.

Fineman, S. (Ed.) (2000). Emotion in Organizations. Sage Publications, London. 
Goleman, D. (1995). Emotional Intelligence - Why it can matter more than IQ. London: Broomsbury Publishing.

Goleman, D. (2001). The Emotionally Intelligent Workplace, San Francisco, CA: Jossey-Bass.

Groves, K. S., McEnrue, M. P. \& Shen, W. (2008). Developing and measuring the emotional intelligence of leaders. Journal of Management Development, 27(2), 225-250.

Kent. T. W. (2006). Leadership and emotions in health care organizations. Journal of Health Organization and Management, 20(1), 49-66.

Kinicki, A., \& Kreitner, R. (2009). Organizational Behavior, (9 ${ }^{\text {th }}$ Ed). U.S., McGraw-Hill, Inc., 137141.

Kouzes, J.M., \& Posner, B.Z. (1995). The Leadership Challenge, Jossey-Bass Inc., San Francisco, CA.

Luthans, F. (2008). Organizational Behaviour. (11th ed.), Singapore: McGraw-Hill International Edition.

Mark, A. (2005). Organizing emotions in health care. Journal of Health Organization and Management, 19(4/5), 277 - 289.

Mayer, J., Salovey, P., \& Caruso, D. (2003). Measuring emotional intelligence with the MSCEIT V2.0, Emotion, 3, 97-105.

McQueen A. (1997). The emotional work of caring, with a focus on gynaecological nursing. Journal of Clinical Nursing, 6, 233-240. McQueen A. (2000) Nurse-patient relationships and partnership in hospital care. Journal of Clinical Nursing, 9, 723-731.

McQueen A. (2000). Nurse-patient relationships and partnership in hospital care. Journal of Clinical Nursing, 9, 723-731.

McQueen, A. (2004). Emotional intelligence in nursing work. Journal of Advanced Nursing, 47(1), 101-108.

Meyer, B., Fletcher, T., \& Parker, S. (2004). Enhancing emotional intelligence in the health care environment: an exploratory study. Health Care Manager, 23(3), 225-34.

Minda, K. (2013). The practice of meditation: a path to maximize the quality of life. Journal of Transpersonal Research, 5(1), 20-35.

Morrison, T. (2007). Emotional intelligence, emotion and social work: Context, characteristics, complications and contribution. British Journal of Social Work, 37(2), 245-263.

Naseer, Z., Chisti, S., Rahman, F., \& Jumani, N. B. (2011). Impact of emotional intelligence on team performance in Higher Education Institutes.International Online Journal of Educational Sciences, 3(1), 30-46.

O’Boyle, E., Humphrey, R.H., Pollack, J.M., Hawver, T.H. \& Story, P. (2011). The Relation Between Emotional Intelligence and Job Performance: A Meta-Analysis. Journal of Organizational Behavior, 32, 788-818.

Palmer, B., Walls, M., Burgess, Z., \& Stough, C. (2001). Emotional intelligence and effective leadership. Leadership \& Organization Development Journal, 22(1), 5-10.

Pescosolido, A. T. (2002). Emergent leaders as managers of group emotion. The Leadership Quarterly, 13, 583-599.

Rahim, S. H., \& Malik, M. I. (2010). Emotional intelligence \& organizational performance:(A case study of banking sector in Pakistan). International Journal of Business and Management, 5(10), p191.

Rahim, M.A., Psenicka, C., Polychroniou, P., Oh, S.Y., Ferdausy, S. \& Dias, J.F. (2006). Emotional intelligence and transformational leadership: a group level analysis in five countries. Current Topics in Management, 11(1), 223-36.

Rosenfeld, P., Giacalone, R.A., \& Riordan, C.A. (1995). Impression management in organizations: Theory, measurement, practice. London: Routledge

Mayer, J. D., \& Salovey, P. (1993). The intelligence of emotional intelligence. Intelligence, 17(4), 433442.

Sarinnapakorn, F. \& Sucaromana, U. (2013). Emotional Intelligence among Business Consultants: A Comparative Study. Asian Social Science, 9(3), 1-6. 
1110

Secker J., Pidd F. \& Parham A. (1999). Mental health training needs of primary health care nurses. Journal of Clinical Nursing, 8(6), 643-652.

Singh, D. (2003). Emotional Intelligence at work, $3^{\text {rd }}$ ed., London: Sage Publications Ltd.

Smith P. (1992). The Emotional Labour of Nursing. Macmillan, Houndmills.

The Journal of Nursing (2007). Emotional Intelligence in the Nursing Profession, 1, American Society of Registered Nurses. Retrieved on 14 June 2013 through http://www.asrn.org/journal-nursing/202emotional-intelligence-in-the-nursing-profession.html

Vitello-Cicciu, J.M. (2002). Exploring emotional intelligence. Implications for nursing leaders. Journal of Nursing Administration, 32(4), 203-210.

Yin, R. K. (2009). Case study research: Design and methods, Applied Social Research Methods Series, ( $4^{\text {th }}$ Ed.), New Delhi: Sage Publications Ltd. 